\title{
Association of age, sex and BMI with the rate of change in tibial cartilage volume: a 10.7-year longitudinal cohort study
}

\author{
Guoqi Cai ${ }^{1+}$ D, Matthew Jiang ${ }^{2+}$, Flavia Cicuttini ${ }^{3}$ and Graeme Jones ${ }^{1 *}$
}

\begin{abstract}
Background: To describe the association of age, sex and body mass index with the rate of change of tibial knee cartilage volume over 10.7 years in a community-based sample of older adults.

Methods: Four hundred and eighty-one participants (49\% female, mean age 60.8 years [range $51.1-79.7$ ], 49\% had knee pain and 58\% radiographic osteoarthritis) were included. Tibial cartilage volume of the right knee was assessed on T1-weighted fat-suppressed 1.5 T MRI at baseline and 10.7 years. Data analyses were performed using linear regression models.

Results: The average rate of loss of cartilage volume was 1.2\%/year (range 0.2-3.9\%) with all participants losing cartilage volume over the study period. There was a significant association between age and loss of tibial cartilage volume in the medial (0.023\%/year, $95 \%$ confidence interval [CI] 0.010 to $0.036 \%, p<0.001)$, lateral $(0.013 \% /$ year, $95 \% \mathrm{Cl} 0.003$ to $0.023 \%, p=0.012)$ and total tibia $(0.018 \% / y e a r, 95 \% \mathrm{Cl} 0.009$ to $0.026 \%, p<0.001)$. Higher body mass index at baseline and increases in body mass index over time were associated with a greater tibial cartilage loss at the medial (body mass index at baseline $0.040 \% / y e a r, 95 \% \mathrm{Cl} 0.022$ to $0.058 \%, p<0.001$; increases in body mass index $0.055 \% / y e a r, 95 \% \mathrm{Cl} 0.018$ to $0.093 \%, p=0.004$ ) but not lateral compartment. No evidence of non-linear relationships was observed. Compared to males, females lost more lateral tibial cartilage with increasing age (0.023\%/year, $95 \% \mathrm{Cl} 0.003$ to $0.043 \%, p=0.024$ for interaction).

Conclusions: Tibial cartilage volume declines at a faster rate with increasing age and body mass index in both males and females, particularly in the medial compartment. In contrast to the low rate of change in radiographs, our findings suggest that cartilage loss at the tibia is universal in this age group.
\end{abstract}

Keywords: Age, Cartilage volume, Magnetic resonance imaging, Osteoarthritis

\section{Background}

Osteoarthritis (OA) is the most common form of arthritis, characterised by gradual loss of articular cartilage [1]. The prevalence of OA increases with age implying that the disease progresses with age. However, current evidence concerning the role of age on the structural progression of $\mathrm{OA}$ is inconsistent.

Radiographic joint space width at the tibiofemoral joint has historically been considered a good measure of change in cartilage volume; however, radiograph-based

\footnotetext{
* Correspondence: Graeme.Jones@utas.edu.au

${ }^{\dagger}$ Guoqi Cai and Matthew Jiang contributed equally to this work.

${ }^{1}$ Menzies Institute for Medical Research, University of Tasmania, Private Bag

23, Hobart, TAS 7001, Australia

Full list of author information is available at the end of the article
}

studies have reported inconsistent findings with regard to joint space or cartilage loss with age [2-7]. While most studies show low rates of progression over time and that this only occurs in some subjects $[2,6,7]$, this may reflect inaccuracies with radiographs over time. Change in knee cartilage volume on magnetic resonance imaging (MRI) correlates poorly with the change in radiographic joint space width [8], which suggests that radiographic change may not be sensitive to cartilage loss. MRI-based cartilage loss has been shown to have greater sensitivity to change [9-12] and is recognised as a valid, accurate and reproducible tool to measure articular cartilage volume [13-15] and its rate of change [16-19]. We have previously reported on age and

(C) The Author(s). 2019 Open Access This article is distributed under the terms of the Creative Commons Attribution 4.0 International License (http://creativecommons.org/licenses/by/4.0/), which permits unrestricted use, distribution, and 
cartilage loss in a younger population suggesting cartilage loss is almost universal after the age of 40 but there are no studies in the elderly [1,20]. MRI-based studies have also demonstrated age to be associated with increased severity and prevalence of cartilage defects [14] as well as cartilage thinning [21]. Moreover, there is a recognised sex difference, with women having a higher prevalence of OA than men, particularly beyond the age of 50 [22], and there are also sex differences in cartilage loss in middle-aged adults [1]. In older adults, cartilage loss may be more likely to vary between sexes due to changes in hormone levels in postmenopausal women, which are associated with progressive articular structural changes [23]. Moreover, a significant sex difference in growth factors has been confirmed in older adults [24]. Importantly, these growth factors including transforming growth factor- $\beta$ and insulin-like growth factor-1 play an important role in cartilage formation and repair [25].

Previous population-based studies have evaluated change in cartilage volume over a relatively short time of only 2 years $[1,16-20]$ showing a deleterious $[1,19,20]$ or no association [16-18] between age and greater cartilage loss. The inconsistent results may be explained by the increased cartilage thickness at early stages of OA due to swelling or softening of cartilage [26-29]; a longer follow-up in older adults would minimise such influence. Moreover, high body mass index (BMI) is a major risk factor for the development and progression of OA [30]. However, there is limited evidence showing the effect of BMI on knee cartilage volume, especially with a long-term follow-up [31]. Gersing et al. [32-34] found that weight loss may slow knee cartilage deterioration in overweight and obese individuals over 48 and 96 months, in which cartilage deterioration was assessed using the modified Whole-Organ Magnetic Resonance Imaging Score (WORMS) [32] or knee cartilage T2 values $[33,34]$. The aim of this study, therefore, was to describe the association of age, sex and BMI with the rate of loss of tibial knee cartilage volume in a population-based sample followed up over a 10.7-year period, which was much longer than previous studies. Moreover, this population-based cohort consisted of older adults (50-80 years), while previous studies have included only middle-aged adults (<60 years) $[1,20]$. We hypothesised that tibial cartilage loss would be greater with increasing age and BMI, especially in females.

\section{Patients and methods}

\section{Study participants}

The Tasmanian Older Adult Cohort (TASOAC) study is an ongoing prospective study in southern Tasmania that began in 2002 [35]. Men and women (98\% Caucasian) aged $50-80$ years old were randomly selected from the electoral roll in southern Tasmania (population 229,
000), a comprehensive population listing using sexstratified random sampling without replacement (response rate 57\%). Participants were excluded if they were institutionalised or had contraindications to MRI. There were no other participation restrictions. The study was approved by the Southern Tasmanian Health and Medical Research Ethics Committee, and written informed consent was obtained from all participants.

Baseline measurements (phase I) were conducted from April 2002 to September 2004. Follow-up data were collected at 2.7 (range 1.7 to 2.9), 5 years (range 4.6 to 5.9) and 10.7 years (range 9.2 to 12.5). A total of 1099 participants were enrolled in this cohort at baseline, and 569 (51.8\%) participated the 10.7-year follow-up. The current study consists of 481 participants who underwent MRI assessments and had validated MRI scans at baseline and the latest (10.7-year) follow-up.

\section{Magnetic resonance imaging}

MRI of the right knee was acquired using a 1.5-T wholebody MRI unit (Picker, Cleveland, OH, USA) at baseline (2002-2004) and another 1.5-T whole-body MRI unit (Siemens, Espree, Pennsylvania, USA) at 10.7 years (2013-2015) due to the decommissioning of the old MRI in 2007. Both MRI units used a standard commercial transmit-receive extremity coil. MRI assessments for each participant were done according to a prespecified protocol. Tibial (medial and lateral) cartilage volume at baseline and 10.7 years follow-up was assessed using T1weighted fat-suppressed three-dimensional gradient recall acquisition in the steady state, flip angle $55^{\circ}$, repetition time $58 \mathrm{msec}$, echo time $12 \mathrm{msec}$, field of view 16 $\mathrm{cm}, 60$ partitions and $512 \times 512$ matrix. Sagittal images were obtained at a partition thickness of $1.5 \mathrm{~mm}$ and an in-plane resolution of $0.31 \times 0.31 \mathrm{~mm}$. The measurements were conducted in a paired fashion with known chronology by a trained reader using OsiriX software for Mac (University of Geneva, Geneva). The volumes of individual cartilage plates (medial tibia and lateral tibia) were isolated from the total volume by manually drawing disarticulation contours around the cartilage boundaries on a section by section basis. These data were then resampled by means of bilinear and cubic interpolation (area of $0.31 \times 0.31 \mathrm{~mm}$ and $1.5 \mathrm{~mm}$ thickness, continuous sections) for the final 3D rendering (Fig. 1). The intra-observer coefficient of variation (CV) for cartilage volume measures was $2.1 \%$ for the medial tibial and $2.2 \%$ for the lateral tibial [15].

Tibial plateau bone area at the medial and tibial compartments was also measured on T1-weighted MRI and defined as the cross-sectional surface area of the tibial plateau. The $\mathrm{CV}$ for intra-observer repeatability was $2.2-2.6 \%[36]$. 

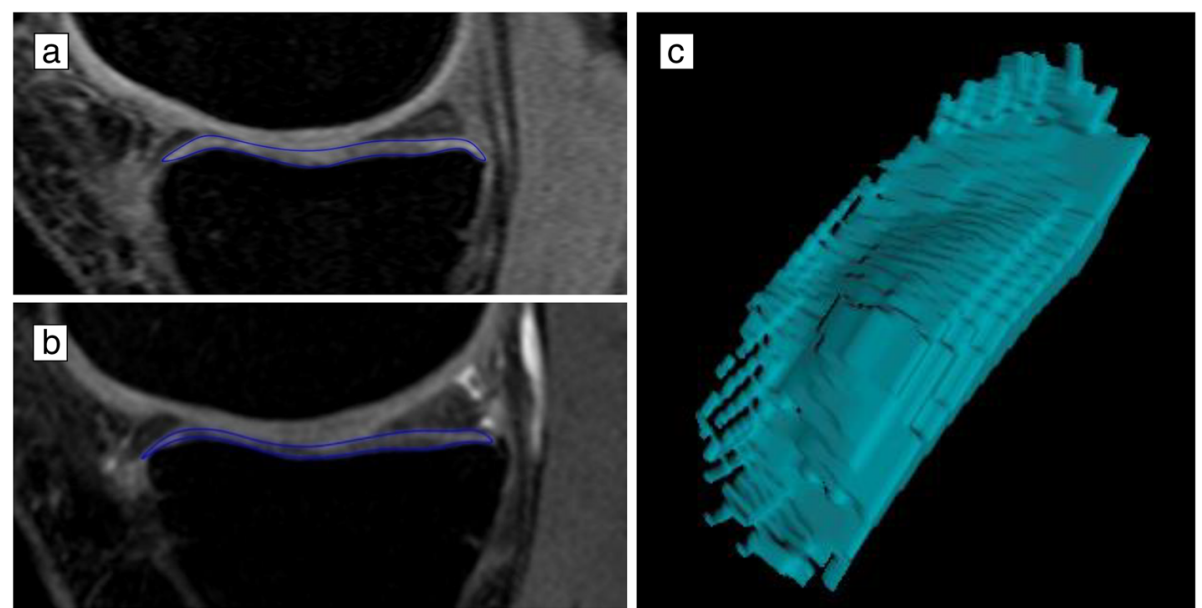

Fig. 1 Examples of change in tibial cartilage volume at baseline (a) and follow-up (b) and 3D representation of tibial cartilage segmentation (c)

\section{Radiographic OA}

A standing anteroposterior semi-flexed view of the right knee with $15^{\circ}$ of fixed knee flexion was performed for all participants at baseline. Joint space narrowing (JSN) and osteophytes were graded on a scale of $0-3$ according to the Osteoarthritis Research Society International (OARSI) atlas [37], and radiographic OA was defined as the presence of any JSN or osteophytes. Severe radiographic OA in this study was defined as having either grade $3 \mathrm{JSN}$ or grade 3 osteophytes at any of the medial and lateral sites.

\section{Clinical symptoms}

At baseline and each follow-up, knee pain was assessed using the Western Ontario and McMaster Universities Osteoarthritis Index (WOMAC) pain subscale with total scores ranging from 0 (no pain) to 45 (worst pain) [38]. Functional disability was assessed using the WOMAC function subscale with total scores ranging from 0 (no disability) to 153 (worst disability). Each item of the WOMAC pain and function subscale was assessed using a $0-9$ numeric rating scale, where 0 indicates no pain or function disability and 9 severe pain or function disability. The presence of knee pain and functional disability was defined as WOMAC pain and function scores larger than 0 , respectively.

\section{Anthropometrics}

Height and weight were measured at the baseline clinic visit, and BMI was calculated by weight divided by height squared $\left(\mathrm{kg} / \mathrm{m}^{2}\right)$. Weight change over 10.7 years was classified as weight loss, weight gain and stable weight with a commonly used cut point of $5 \mathrm{~kg}$ [39].

\section{Physical activity}

Physical activity was evaluated as steps/day using pedometer (Omron HJ-003 and HJ-102, Omron Healthcare,
Kyoto, Japan) at baseline, 2.7- and 5-year follow-up. Each participant was instructed to wear a pedometer for seven consecutive days. This was repeated 6 months later to account for seasonal variation. Mean steps/day was calculated as the average of the days worn at both time points [40].

\section{Other measures}

Participants were asked if they had ever been diagnosed by a doctor as having rheumatoid arthritis at baseline. History of knee surgery (other than knee replacement) was recorded at baseline while history of knee injury was evaluated at the 2.7-year follow-up. Moreover, new incidents of knee surgery were assessed during the course of the study by asking "since your last interview, have you had any knee surgery?". New knee injury was not recorded.

\section{Statistical analysis}

Baseline characteristics were shown as percentage, mean (standard deviation [SD]) or median (interquartile range [IQR]) as appropriate. The characteristics of age groups (group 1, 50-60 years; group 2, 60-70 years; and group $3,70-80$ years) were compared using chi-square tests, analysis of variance or the Kruskal-Wallis test. We calculated both absolute $\left(\mathrm{mm}^{3} /\right.$ year $)$ and percentage loss (\%/year) of cartilage volume per annum, which were calculated as (a) absolute loss per annum $=$ (baseline cartilage volume - follow-up cartilage volume)/time between two scans in years, and (b) percentage loss per annum = $100 \times$ [(baseline cartilage volume-follow-up cartilage volume)/baseline cartilage volume]/time between two scans in years.

Linear regression analysis was performed to assess the association of age and BMI with loss of cartilage volume from baseline to 10.7 years with adjustment for potential 
confounders at baseline (sex, BMI, radiographic OA, history of knee surgery/injury, physical activity and sitespecific tibial cartilage volume). The estimated coefficients with 95\% confidence intervals were presented for loss of cartilage volume per 1 year older in age or one unit higher in BMI. In addition, non-linear associations of age and BMI with tibial cartilage loss were assessed by using locally weighted regression smoothing. Cartilage loss among different age groups (i.e. group 1, 50-60 years; group 2, 60-70 years; and group 3, 70-80 years) and groups of weight change (i.e. group 1, weight loss > $5 \mathrm{~kg}$; group 2, weight change $<5 \mathrm{~kg}$; group 3 , weight gain $>5 \mathrm{~kg}$ ) was also compared in the regression models. Because of the potential sex differences for the association between age and cartilage volume loss from previous evidence [1], stratified analysis by sex was prespecified. Despite this, we checked the interaction between age and sex for the annual loss of cartilage volume by adding an age $\times$ sex term in regression models. Moreover, the interactions between each of age and BMI with the presence and absence of radiographic OA (i.e. age $\times$ radiographic $\mathrm{OA}$ and $\mathrm{BMI} \times$ radiographic $\mathrm{OA}$, respectively) and between BMI and sex (i.e. BMI $\times$ sex) were assessed, but no significant interactions were found.

Four sensitivity analyses were conducted in this study. First, to address the missing data ( 0.2 to $8.1 \%$ missing) of covariates, multiple imputations were carried out. Twenty imputations were performed using baseline variables with complete data assuming missing at random. Baseline characteristics of participants included in and excluded from the study were compared. Second, participants were excluded if they had either severe radiographic OA or rheumatoid arthritis at baseline, considering the potential impact of severe radiographic OA and comorbidity of rheumatoid arthritis on the progression of cartilage loss. Third, the association between age and tibial cartilage loss was conducted by further adjusting for the site-specific tibial bone size and change in BMI, given that evidence has shown a potential effect of them on cartilage volume loss [41, 42]. Moreover, having new surgeries in the knee (not knee replacement) during the course of the study was also adjusted for as the fourth sensitivity analysis.

All statistical analyses were performed using Stata (version 15.1, StataCorp, TX, USA). Statistical significance was set at a $p$ value of $\leq 0.05$ (two-tailed).

\section{Results}

\section{Participants}

A total of 481 participants ( $49 \%$ female, mean age $60.8 \pm$ 6.3 years [range 51.1-79.7]) had paired MRI imaging at baseline and 10.7 years. Baseline characteristics between participants were split by 3 age groups (Table 1). There were no significant differences in sex, BMI, knee pain and function scores; tibial bone size; and the prevalence of radiographic OA (including JSN and osteophytes) among groups. Tibial cartilage volume $\left(\mathrm{mm}^{3}\right)$ at baseline was smaller in the oldest age group (aged 70-80 years), and physical activity (steps/day) was reduced with increasing age.

\section{Age and tibial cartilage volume loss}

The average rate of loss of total tibial cartilage volume was $1.2 \%$ per annum (range 0.2 to $3.9 \%$ ) with $100 \%$ of participants losing tibial cartilage volume over the study period. There was a positive correlation between age and loss of tibial cartilage volume, with older adults losing more tibial cartilage volume per year (\%/year, Fig. 2a); similar results were found for the correlation between BMI at baseline and loss of tibial cartilage volume (\%/year, Fig. 2b). No evidence of a non-linear association between age or BMI and tibial cartilage loss (\%/year) was observed (Fig. 2c, d). Additional file 1: Figure S1 shows the association of age and BMI with absolute loss of tibial cartilage volume per year $\left(\mathrm{mm}^{3} /\right.$ year). In multivariable analysis (Table 2), age was significantly associated with loss of tibial cartilage volume at both the medial and lateral compartments, independent of radiographic OA. There was a significant interaction between age and sex for lateral tibial cartilage volume loss. In stratified analysis, females lost more cartilage from all compartments with increasing age; however, the association for males was only significant for medial and total tibial cartilage loss.

Comparing participants by age groups (Fig. 3 and Additional file 1: Figure S2), there was a significant trend that older participants lost more tibial cartilage volume at both medial and lateral compartments. These results persisted after further adjustment for potential confounders (Table 3). Of note, there was a trend to a significant interaction between age group and sex for loss of lateral tibial cartilage $(p=0.084)$, and females lost more lateral tibial cartilage volume with increasing age compared to males. No significant interaction between age group and sex was observed for cartilage loss at the medial or total tibia. In prespecified stratified analysis, females lost more medial and lateral cartilage volume with increasing age group, but in males, there was a significantly greater medial and total tibial cartilage loss with increasing age group and no association for cartilage loss at the lateral compartment.

\section{Body mass index and tibial cartilage volume loss}

There was a significant association between BMI at baseline and loss of cartilage volume at the medial but not lateral compartment. After adding change in BMI over time to the model, both BMI at baseline and change in BMI were associated with a greater loss of medial tibial 
Table 1 Characteristics of study populationt

\begin{tabular}{|c|c|c|c|c|}
\hline & Age $50-60$ years $(n=256)$ & Age $60-70$ years $(n=181)$ & Age 70-80 years $(n=44)$ & $p$ value $^{\ddagger}$ \\
\hline Age (year) & $55.9(2.3)$ & $64.7(3.0)$ & $73.3(2.3)$ & $<0.001$ \\
\hline Females, \% & 52 & 48 & 45 & 0.604 \\
\hline $\mathrm{BMI}\left(\mathrm{kg} / \mathrm{m}^{2}\right)$ & $27.9(4.8)$ & $27.4(3.9)$ & $27.6(3.9)$ & 0.580 \\
\hline Radiographic OA, \% & 56 & 59 & 72 & 0.170 \\
\hline Joint space narrowing & 56 & 58 & 72 & 0.173 \\
\hline Osteophytes & 6 & 11 & 5 & 0.128 \\
\hline Tibial bone size $\left(\mathrm{mm}^{2}\right)$ & $3279.9(469.5)$ & $3366.6(498.0)$ & $3382.1(558.5)$ & 0.190 \\
\hline WOMAC pain score (0-45), median (IQR) & 0.5 (0 to 4$)$ & 0 (0 to 3 ) & $1(0$ to 4$)$ & 0.541 \\
\hline Any pain, \% & 50 & 46 & 52 & 0.612 \\
\hline WOMAC function score (0-153), median (IQR) & $0(0$ to 8.5$)$ & $1(0$ to 7$)$ & $4.5(0$ to 16$)$ & 0.095 \\
\hline Any functional disability, $\%$ & 49 & 53 & 68 & 0.057 \\
\hline History of knee surgery, \% & 11 & 9 & 7 & 0.741 \\
\hline History of knee injury, \%* & 14 & 10 & 9 & 0.413 \\
\hline Physical activity (steps/day) & $9639.8(3239.9)$ & $9172.3(3128.9)$ & $7625.0(2803.0)$ & $<0.001$ \\
\hline \multicolumn{5}{|l|}{ Cartilage volume $\left(\mathrm{mm}^{3}\right)$} \\
\hline Medial tibial & $1509.5(398.1)$ & $1523.8(449.3)$ & $1438.6(397.0)$ & 0.479 \\
\hline Lateral tibial & $2096.4(591.5)$ & $2046.9(651.5)$ & $1803.2(599.9)$ & 0.015 \\
\hline Total tibial & $3606.0(917.1)$ & 3570.7 (1018.6) & $3241.8(907.5)$ & 0.065 \\
\hline
\end{tabular}

$B M I$ body mass index, IQR interquartile range, OA osteoarthritis, WOMAC Western Ontario and McMaster Universities Osteoarthritis Index

${ }^{\dagger}$ Data are presented as mean (standard deviation) unless specified otherwise (e.g. percentage, median (IQR)). Italicised data denotes statistically significant result

${ }^{\ddagger} p$ values are calculated using chi-square tests, analysis of variance or the Kruskal-Wallis test

*History of knee injury was assessed at the 2.7 -year follow-up

cartilage volume (Table 4). Moreover, there was a significant association between weight change (by categories) and medial tibial cartilage loss in multivariable analysis (Fig. 4 and Additional file 1: Figure S3).

\section{Sensitivity analysis}

In sensitivity analysis, multiple imputations for missing data produced similar results, except that the association between age group and loss of medial and total tibial cartilage volume became statistically significant in males (Additional file 1: Table S1-S3). Baseline characteristics of participants included in the study were similar to those who were not, although participants included were younger and had less knee pain and functional disability (Additional file 1: Table S4). The association between age or age group and loss of tibial cartilage volume was attenuated in females, after excluding participants who had rheumatoid arthritis $(n=39,8.1 \%)$ and/or severe radiographic OA $(n=10,2.1 \%)$ at baseline (Additional file 1: Table S5-S7). In multivariable analysis, further adjustment of site-specific bone size at baseline and change in BMI or new surgeries in the knee during the follow-up did not change the association between age or BMI and cartilage loss (data not shown).

\section{Discussion}

In this 10.7-year longitudinal study of a populationbased cohort, all participants lost tibial knee cartilage over time and increasing age was associated with a greater loss of tibial cartilage volume. In addition, the annual loss of cartilage volume was faster in females, particularly in the lateral compartment. Moreover, both BMI at baseline and increase in BMI over time were associated with a greater loss of tibial cartilage volume at the medial but not lateral compartment. The findings of this study reveal that tibial cartilage loss is universal in older adults and will become faster over time, particularly in those with higher BMI at baseline and increased BMI over time.

The results of this study contrast to reports based on radiographic findings where less than $50 \%$ of the older population have progressive joint space narrowing over 4 to 14 years [2-7]. This most likely reflects the higher sensitivity of MRI to change. Moreover, we found that cartilage loss appeared to increase with increasing age. This finding is consistent with two previous radiographic studies conducted in a similar [2] or younger [3] population but contrasts to others $[4,7]$ that suggested a similar rate of change in radiographs among age groups. The inconsistency is most likely due to that radiographic joint space is only a surrogate measure of articular cartilage thickness, such that a small change in knee cartilage volume may not be captured in radiographs. Furthermore, radiographic joint space width can be substantially influenced by the position of the knee and meniscal pathology [43], leading to misclassification. 


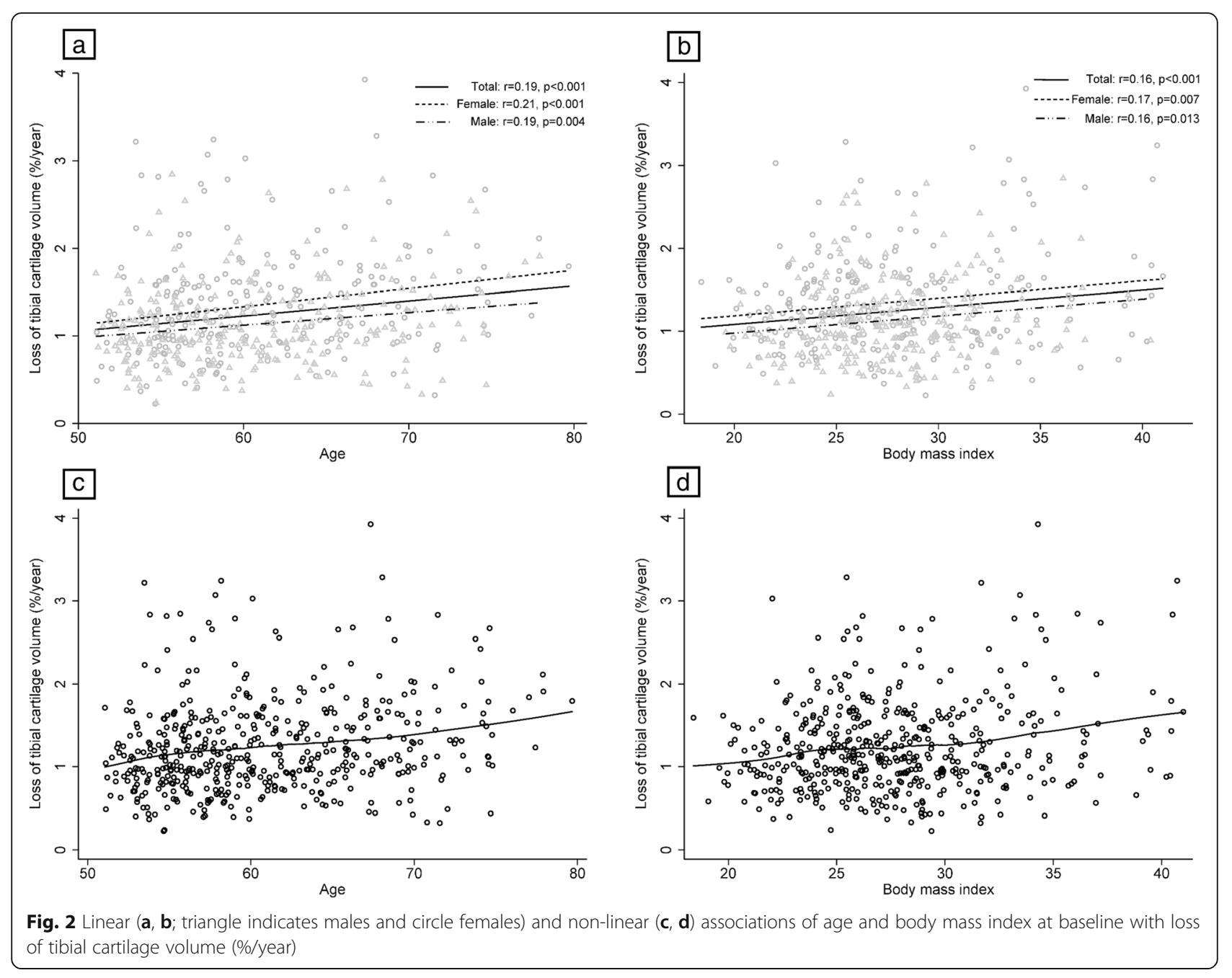

Table 2 Association of age and sex with tibial cartilage volume change over 10.7 years

\begin{tabular}{|c|c|c|c|c|c|}
\hline & \multicolumn{3}{|c|}{ Multivariable, $\beta$ (95\% Cl) } & \multirow{2}{*}{$\begin{array}{l}\text { Interaction with sex (male } \\
\text { vs. female) }\end{array}$} & \multirow{2}{*}{$\begin{array}{l}p \text { for } \\
\text { interaction }\end{array}$} \\
\hline & Females $(n=211)^{\dagger}$ & Males $(n=217)^{\dagger}$ & Combined $(n=428)^{\ddagger}$ & & \\
\hline \multicolumn{6}{|c|}{ Loss of cartilage volume ( $\mathrm{mm}^{3} /$ year) } \\
\hline Medial tibial & 0.27 (0.00 to 0.54$)$ & 0.43 (0.14 to 0.73 ) & 0.36 (0.16 to 0.57$)$ & $0.16(-0.24$ to 0.57$)$ & 0.430 \\
\hline Lateral tibial & 0.37 (0.11 to 0.63$)$ & $0.04(-0.27$ to 0.34$)$ & $0.18(-0.02$ to 0.38$)$ & $-0.33(-0.73$ to 0.07$)$ & 0.107 \\
\hline Total tibial & $0.66(0.24$ to 1.07$)$ & 0.49 (0.05 to 0.92$)$ & $0.56(0.26$ to 0.86$)$ & $-0.17(-0.78$ to 0.43$)$ & 0.575 \\
\hline \multicolumn{6}{|c|}{ Loss of cartilage volume (\%/year) } \\
\hline Medial tibial & 0.023 (0.003 to 0.043$)$ & $0.023(0.006$ to 0.041$)$ & 0.023 (0.010 to 0.036$)$ & $0.000(-0.026$ to 0.027$)$ & 0.980 \\
\hline Lateral tibial & 0.025 (0.009 to 0.041$)$ & $0.002(-0.010$ to 0.015$)$ & 0.013 (0.003 to 0.023$)$ & $-0.023(-0.043$ to -0.003$)$ & 0.024 \\
\hline Total tibial & 0.024 (0.010 to 0.038$)$ & $0.012(0.002$ to 0.022$)$ & $0.018(0.009$ to 0.026$)$ & $-0.012(-0.029$ to 0.005$)$ & 0.173 \\
\hline
\end{tabular}

${ }^{\dagger}$ Model 1: adjusted for body mass index, radiographic osteoarthritis, history of knee surgery and knee injury, physical activity and site-specific tibial cartilage volume at baseline

${ }^{\ddagger}$ Model $1+$ sex

Italicised data denotes statistically significant result 


\section{a: Overall}

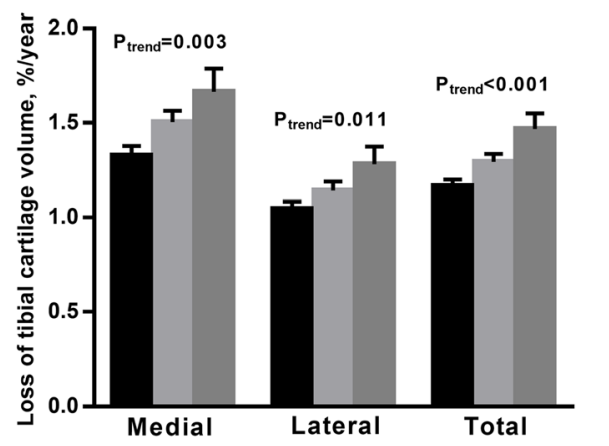

Age $(50-60 \mathrm{y})$

Age $(60-70 y)$

Age $(70-80 \mathrm{y})$

\section{b: Male}

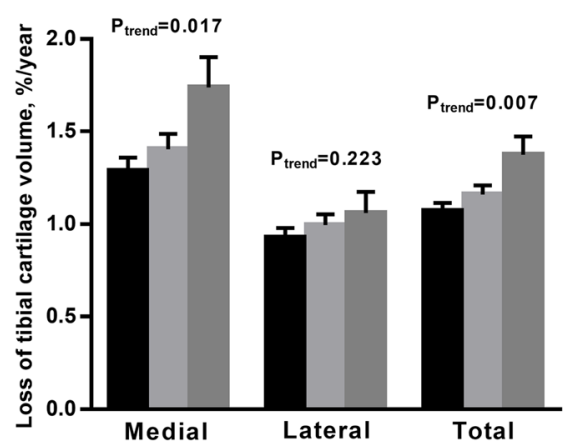

C: Female

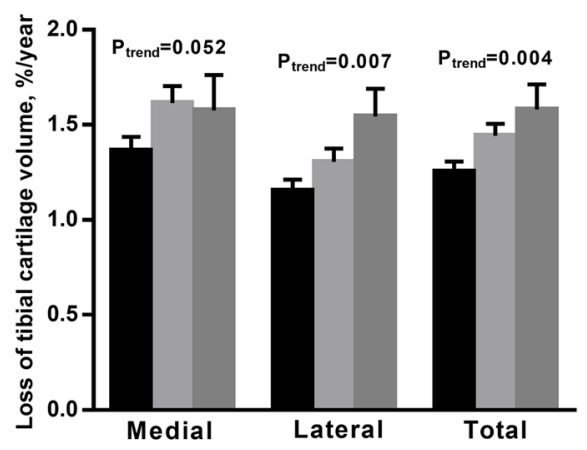

Fig. 3 Loss of tibial cartilage volume among age groups over 10.7 years overall (a) and in males (b) and females (c). Bar graph indicates the mean value of tibial cartilage loss (\%/year), and error bars indicate standard errors. $p$ for trend was calculated by univariable linear regression models

In this population-based older adult cohort, we observed that over a 10.7-year period, the older the subject, the greater the cartilage volume loss implying that annual cartilage loss is not constant but increases over time. Similarly, in another older cohort of OA patients, older patients at baseline experienced a greater loss of tibial cartilage volume over 2 years [19]. Moreover, this association of age with an increased rate of cartilage volume loss has been demonstrated over a 2-year period in a younger population-based cohort with a maximum age of 60 (mean age 45 years vs. 60.8 years in the current study) [1]. The rate of tibial cartilage volume loss was lower in our cohort at $-1.2 \%$ per annum compared to the younger cohort (-3.0\% per annum), but half of the younger cohort was selected based on family risk of osteoarthritis, and these offspring of OA patients have a higher rate of cartilage loss [44] which most likely contributes to the higher rates demonstrated in the younger cohort. In addition, the longer follow-up of our cohort may provide a more reliable representation of the real rate of cartilage loss over time. Indeed, several longitudinal studies with a short- or medium-term follow-up have observed an increased cartilage thickness at the medial site of tibiofemoral joint and hypothesised that cartilage swelling and softening prior to MRI-detected cartilage loss may play a role [28, 29]. Moreover, one study found cartilage thickening at the medial femur and cartilage thinning at trochlea of the femur [29], indicating a variation of cartilage change among anatomic sites.

Females appeared to lose cartilage at a faster rate than men, especially in the lateral compartment. With adjustment for other factors including cartilage volume at baseline, females lost cartilage at a faster rate with age in both the medial and lateral compartments, whereas males did not appear to lose much cartilage from the lateral tibial compartment. The sex difference in lateral tibial cartilage volume loss may be related to the anatomical variations between knees in men and women. Women have been shown to have a greater valgus knee angle [45] compared to men, which is associated with an increased risk of lateral compartment cartilage loss [46, 47]. A higher prevalence of OA [22, 48], cartilage defects [49] and rate of cartilage loss [1] in women has been reported in other studies. It may reflect a lower baseline cartilage volume in females as well as an increased rate of cartilage loss in postmenopausal females [15]. When divided by age groups, the age association for females was statistically significant for both medial and lateral 
Table 3 Association of age group and sex with loss of tibial cartilage volume (\%/year) over 10.7 years

\begin{tabular}{|c|c|c|c|}
\hline & \multicolumn{3}{|l|}{ Multivariable, $\beta$ (95\% Cl) } \\
\hline & Medial & Lateral & Total tibia \\
\hline \multicolumn{4}{|l|}{ Combined $(n=428)^{\dagger}$} \\
\hline Age $50-60$ years & Ref. & Ref. & Ref. \\
\hline Age 60-70 years & $0.16(-0.01$ to 0.33$)$ & $0.07(-0.05$ to 0.20$)$ & 0.11 (0.00 to 0.22 ) \\
\hline Age $70-80$ years & 0.38 (0.08 to 0.67$)$ & 0.31 (0.08 to 0.53) & 0.35 (0.16 to 0.54$)$ \\
\hline$p$ for trend & 0.006 & 0.012 & $<0.001$ \\
\hline \multicolumn{4}{|l|}{ Females $(n=211)^{\ddagger}$} \\
\hline Age $50-60$ years & Ref. & Ref. & Ref. \\
\hline Age 60-70 years & 0.26 (0.01 to 0.51$)$ & $0.11(-0.09$ to 0.31$)$ & $0.17(-0.01$ to 0.34$)$ \\
\hline Age $70-80$ years & $0.29(-0.19$ to 0.76$)$ & 0.60 (0.22 to 0.98$)$ & $0.47(0.13$ to 0.80$)$ \\
\hline$p$ for trend & 0.042 & 0.007 & 0.004 \\
\hline \multicolumn{4}{|l|}{ Males $(n=217)^{\ddagger}$} \\
\hline Age $50-60$ years & Ref. & Ref. & Ref. \\
\hline Age 60-70 years & $0.09(-0.14$ to 0.32$)$ & $0.02(-0.14$ to 0.18$)$ & $0.05(-0.08$ to 0.19$)$ \\
\hline Age $70-80$ years & 0.41 (0.03 to 0.79$)$ & $0.12(-0.15$ to 0.39$)$ & 0.27 (0.05 to 0.49$)$ \\
\hline$p$ for trend & 0.053 & 0.449 & 0.035 \\
\hline Interaction with sex & $-0.04(-0.29$ to 0.21$)$ & $-0.17(-0.36$ to 0.02$)$ & $-0.10(-0.26$ to 0.07$)$ \\
\hline$p$ for interaction & 0.778 & 0.084 & 0.241 \\
\hline
\end{tabular}

${ }^{\dagger}$ Model 1: adjusted for sex, BMI, radiographic osteoarthritis, history of knee surgery and knee injury, physical activity and site-specific tibial cartilage volume at baseline

${ }^{\ddagger}$ Model 2: adjusted for BMI, radiographic osteoarthritis, history of knee surgery and knee injury, physical activity and site-specific tibial cartilage volume at baseline Italicised data denotes statistically significant results

compartments, which may reflect this postmenopausal increase in the rate of cartilage loss. Moreover, it is important to note that after excluding participants with either rheumatoid arthritis or severe radiographic OA at baseline, the association between age and tibial cartilage loss in females was attenuated (excluding severe radiographic OA only did not lead to these changes). This may suggest that the increased tibial cartilage loss with ageing in females was partly driven by the effect of rheumatoid arthritis on bone and cartilage damage [50]. However, this needs to be confirmed in future studies given that data on rheumatoid arthritis were selfreported and the association between age and cartilage loss was not changed in males.

This study indicated that higher BMI at baseline was a risk factor of structural OA progression over 10.7 years,

Table 4 Association of body mass index and change in body mass index with loss of tibial cartilage volume over 10.7 years $(n=$ 428)

\begin{tabular}{|c|c|c|c|}
\hline & \multirow{2}{*}{$\begin{array}{l}\text { Multivariable, } \beta(95 \% \mathrm{Cl}) \\
\text { BMI at baseline }^{\dagger}\end{array}$} & \multicolumn{2}{|l|}{ Multivariable, $\beta$ (95\% Cl) } \\
\hline & & $\overline{\mathrm{BMI}}$ at baseline ${ }^{\ddagger}$ & Change in $\mathrm{BMI}^{\ddagger}$ \\
\hline \multicolumn{4}{|c|}{ Loss of cartilage volume ( $\mathrm{mm}^{3} /$ year) } \\
\hline Medial tibial & $0.55(0.27$ to 0.84$)$ & $0.58(0.29$ to 0.86$)$ & 0.85 (0.26 to 1.43$)$ \\
\hline Lateral tibial & $0.12(-0.15$ to 0.40$)$ & $0.14(-0.14$ to 0.42$)$ & $0.38(-0.20$ to 0.95$)$ \\
\hline Total tibial & $0.69(0.27$ to 1.12$)$ & $0.73(0.31$ to 1.15$)$ & 1.20 (0.33 to 2.07) \\
\hline \multicolumn{4}{|c|}{ Loss of cartilage volume (\%/year) } \\
\hline Medial tibial & $0.038(0.020$ to 0.056$)$ & $0.040(0.022$ to 0.058$)$ & 0.055 (0.018 to 0.093) \\
\hline Lateral tibial & $0.008(-0.005$ to 0.022$)$ & $0.009(-0.005$ to 0.023$)$ & $0.022(-0.007$ to 0.050$)$ \\
\hline Total tibial & $0.022(0.011$ to 0.034$)$ & $0.023(0.012$ to 0.035$)$ & 0.035 (0.010 to 0.059$)$ \\
\hline
\end{tabular}

${ }^{\dagger}$ Model 1: adjusted for age, sex, radiographic osteoarthritis, history of knee surgery and knee injury, physical activity and site-specific tibial cartilage volume at baseline

${ }^{\ddagger}$ Model 1 + change in BMI over 10.7 years

Italicised data denotes statistically significant results. BMI body mass index, $\mathrm{Cl}$ confidence interval 


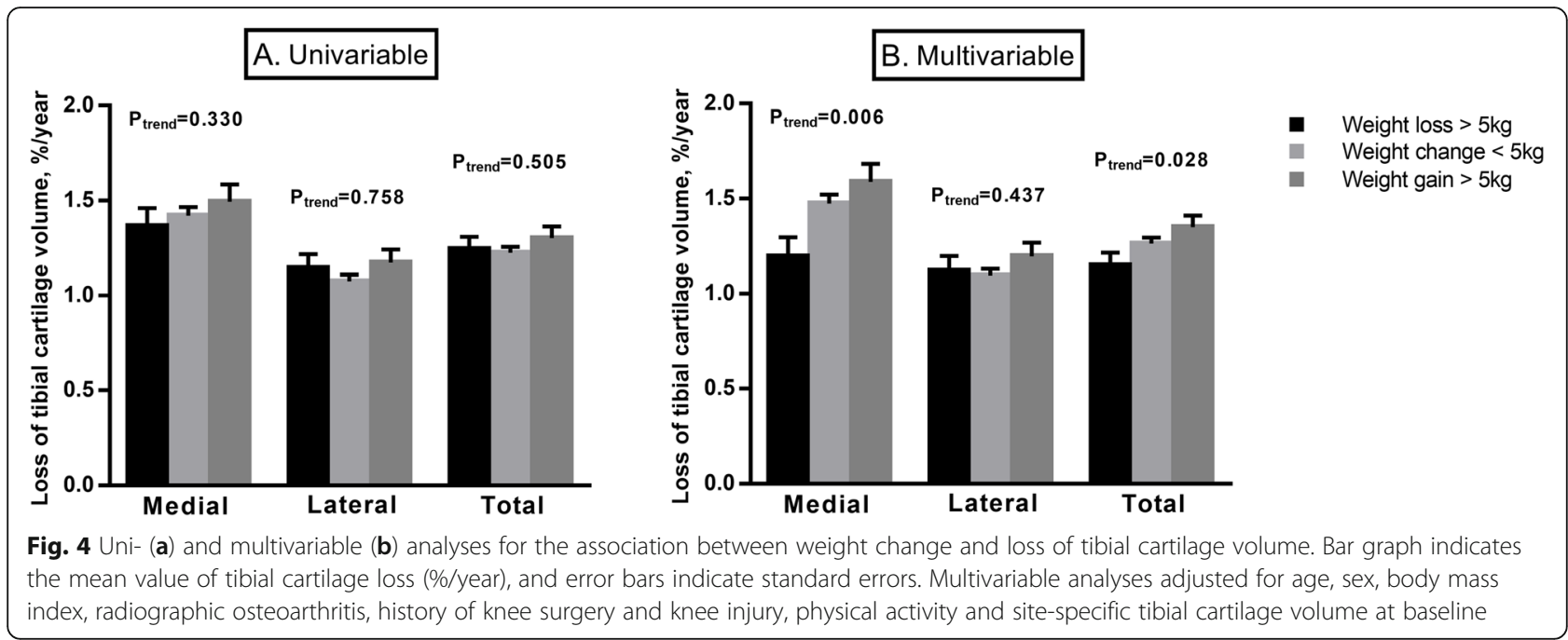

showing a linear relationship with tibial cartilage volume loss in both males and females. Moreover, we found an association between change in BMI and loss of tibial cartilage volume at the medial but not tibial compartment, independent of age and BMI at baseline. In addition, there was a significant association between weight change and medial tibial cartilage volume loss. This agrees with previous studies demonstrating that weight change was associated with loss of tibial cartilage volume at the medial but not lateral compartment in overweight and obese adults over 2.3 to 8 years [32-34, 41, 51]. It remains unclear why a significant association between change in BMI or weight and tibial cartilage loss was only observed at the medial compartment. One potential explanation is that the medial compartment undertakes greater weight-bearing loads and has a higher rate of cartilage loss (as observed in this study); therefore, any effect of weight change on cartilage would be greater at the medial compartment [51]. Moreover, varus knee would also increase the loading to the medial compartment, but knee alignment was not measured in this cohort and this cannot be assessed.

The main strength of our study is the long follow-up of a community-based cohort which is likely to be representative of the general population. To our knowledge, this is the longest follow-up cohort looking at the rate of change in knee cartilage volume as measured by MRI. There are several limitations in this study. First, we did not measure femoral cartilage volume. However, Cicuttini et al. [52] found a strong correlation between longitudinal changes in tibial and femoral cartilage volume $(r=0.81$ and 0.71 in the medial and lateral sites, respectively) in a similar population (mean age 63.7 years, $58 \%$ female) and they suggested that measuring tibial cartilage alone may be adequate to evaluate structural change in the tibiofemoral joint. Second, the known chronology for the measurements of tibial cartilage volume may have introduced an observer-expectancy bias since loss of tibial cartilage volume was found in all participants. Nonetheless, it has been shown that scoring without known chronology substantially decreases sensitivity in detecting clinically relevant changes [53, 54]. In addition, the known chronology for MRI readings is unlikely to bias the association of age, sex and BMI with tibial cartilage loss given that demographic information of study participants was not available to the MRI reader. Third, cartilage volume was measured in only $43.8 \%(481 / 1099)$ of participants in the TASOAC study for the 10.7-year follow up, suggesting a potential attrition bias. While baseline characteristics such as sex, BMI and radiographic OA were similar between included participants and those who were lost to follow-up, included participants were younger (60.8 vs. 64.7 years), were physically more active and had milder knee symptoms at baseline and a lower rate of history of knee surgery, indicating that the study may represent a slightly younger and healthier population. Lastly, tibial cartilage volume was measured at only two time points in this study, and this prevented us from investigating the course of tibial cartilage volume. While our study suggested a linear increase in tibial cartilage loss over time (ageing), this finding needs to be confirmed in studies with MRI measures at multiple time points.

\section{Conclusion}

Knee cartilage volume declines at a faster rate with increasing age and BMI in both males and females, particularly in the medial compartment. In contrast to the slow rate of change in radiographs, our findings suggest that cartilage loss at the knee is universal in this age group. 


\section{Supplementary information}

Supplementary information accompanies this paper at https://doi.org/10. 1186/s13075-019-2063-z.

Additional file 1 : Table S1. Association of age and sex with loss of tibial cartilage volume over 10.7 years after multiple imputations for missing data. Table S2. Association between age group and loss of tibial cartilage volume (\%/year) over 10.7 years after multiple imputations for missing data. Table S3. Association of body mass index and change in body mass index with loss of tibial cartilage volume over 10.7 years after multiple imputations for missing data. Table S4. Characteristics of participants included in and excluded from the study. Table S5. Association of age and sex with loss of tibial cartilage volume over 10.7 years after excluding participants with rheumatoid arthritis or severe radiographic osteoarthritis. Table S6. Association between age group and loss of tibial cartilage volume (\%/year) over 10.7 years after excluding participants with rheumatoid arthritis or severe radiographic osteoarthritis. Table S7. Association of body mass index and change in body mass index with loss of tibial cartilage volume over 10.7 years after excluding participants with rheumatoid arthritis or severe radiographic osteoarthritis. Figure S1. Linear (A and B, triangle indicates males and circle females) and non-linear ( $C$ and $D)$ associations of age and body mass index at baseline with loss of tibial cartilage volume $\left(\mathrm{mm}^{3} /\right.$ year). Figure S2. Loss of tibial cartilage volume among age groups over 10.7 years overall (A) and in males (B) and females (C). Figure S3. Uni- (A) and multivariable (B) analyses for the association between weight change and loss of tibial cartilage volume.

\section{Abbreviations}

BMI: Body mass index; CV: Coefficient of variation; IQR: Interquartile range; JSN: Joint space narrowing; MRI: Magnetic resonance imaging;

OA: Osteoarthritis; OARSI: The Osteoarthritis Research Society International; SD: Standard deviation; TASOAC: The Tasmania Older Adult Cohort; WOMAC: The Western Ontario and McMaster Universities Osteoarthritis Index

\section{Acknowledgements}

The authors thank all participants, staffs and volunteers who made this study possible.

\section{Authors' contributions}

GC had full access to all the data in the study and takes responsibility for the integrity of the data and the accuracy of the data analysis. GC and GJ contributed to the study design and acquisition of data. MJ, GC, FC and GJ contributed to the analysis and interpretation of data. MJ, GC, FC and GJ contributed to the manuscript preparation and approval.

\section{Funding}

The Tasmania Older Adult Cohort was funded by the National Health and Medical Research Council of Australia (302204), the Tasmanian Community Fund (D0015018), the Arthritis Foundation of Australia (MRI06161) and the University of Tasmania Institutional Research Grants Scheme (D0015019).

\section{Availability of data and materials}

All data generated or analysed during this study are included in this published article.

\section{Ethics approval and consent to participate}

The study was approved by the Southern Tasmanian Health and Medical Research Ethics Committee, and written informed consent was obtained from all participants.

\section{Consent for publication}

Not applicable.

\section{Competing interests}

The authors declare that they have no competing interests.

\section{Author details}

${ }^{1}$ Menzies Institute for Medical Research, University of Tasmania, Private Bag 23, Hobart, TAS 7001, Australia. ${ }^{2}$ Department of Rheumatology, Royal Hobart
Hospital, Hobart, TAS, Australia. ${ }^{3}$ Department of Epidemiology and Preventive Medicine, Monash University Medical School, Melbourne, Australia.

Received: 18 September 2019 Accepted: 13 November 2019 Published online: 09 December 2019

\section{References}

1. Ding C, Cicuttini F, Blizzard L, Scott F, Jones G. A longitudinal study of the effect of sex and age on rate of change in knee cartilage volume in adults. Rheumatology (Oxford). 2007;46(2):273-9.

2. Leyland KM, Hart DJ, Javaid MK, Judge A, Kiran A, Soni A, Goulston LM, Cooper C, Spector TD, Arden NK. The natural history of radiographic knee osteoarthritis: a fourteen-year population-based cohort study. Arthritis Rheum. 2012;64(7):2243-51.

3. Schouten JS, van den Ouweland FA, Valkenburg HA. A 12 year follow up study in the general population on prognostic factors of cartilage loss in osteoarthritis of the knee. Ann Rheum Dis. 1992;51(8):932-7.

4. Hart DJ, Doyle DV, Spector TD. Incidence and risk factors for radiographic knee osteoarthritis in middle-aged women: the Chingford study. Arthritis Rheum. 1999;42(1):17-24.

5. Spector TD, Dacre JE, Harris PA, Huskisson EC. Radiological progression of osteoarthritis: an 11 year follow up study of the knee. Ann Rheum Dis. 1992; 51(10):1107-10

6. Pavelka K, Gatterova J, Altman RD. Radiographic progression of knee osteoarthritis in a Czech cohort. Clin Exp Rheumatol. 2000;18(4):473-7.

7. Felson DT, Zhang Y, Hannan MT, Naimark A, Weissman BN, Aliabadi P, Levy D. The incidence and natural history of knee osteoarthritis in the elderly. The Framingham Osteoarthritis Study. Arthritis Rheum. 1995;38(10):1500-5.

8. Hall J, Laslett LL, Martel-Pelletier J, Pelletier JP, Abram F, Ding CH, Cicuttini FM, Jones $\mathrm{G}$. Change in knee structure and change in tibiofemoral joint space width: a five year longitudinal population-based study. BMC Musculoskelet Disord. 2016;17:25.

9. Duryea J, Neumann G, Niu J, Totterman S, Tamez J, Dabrowski C, Le Graverand MP, Luchi M, Beals CR, Hunter DJ. Comparison of radiographic joint space width with magnetic resonance imaging cartilage morphometry: analysis of longitudinal data from the Osteoarthritis Initiative. Arthritis Care Res. 2010;62(7):932-7.

10. Hunter DJ, Zhang W, Conaghan PG, Hirko K, Menashe L, Reichmann WM, Losina E. Responsiveness and reliability of MRI in knee osteoarthritis: a metaanalysis of published evidence. Osteoarthr Cartil. 2011;19(5):589-605.

11. Aitken D, Ding C, Pelletier JP, Martel-Pelletier J, Cicuttini F, Jones G. Responsiveness of magnetic resonance imaging-derived measures over 2.7 years. J Rheumatol. 2014;41(10):2060-7.

12. Reichmann WM, Maillefert JF, Hunter DJ, Katz JN, Conaghan PG, Losina E. Responsiveness to change and reliability of measurement of radiographic joint space width in osteoarthritis of the knee: a systematic review. Osteoarthr Cartil. 2011;19(5):550-6.

13. Jones $\mathrm{G}$, Glisson M, Hynes K, Cicuttini F. Sex and site differences in cartilage development: a possible explanation for variations in knee osteoarthritis in later life. Arthritis Rheum. 2000:43(11):2543-9.

14. Ding C, Cicuttini F, Scott F, Glisson M, Jones G. Sex differences in knee cartilage volume in adults: role of body and bone size, age and physical activity. Rheumatology (Oxford). 2003;42(11):1317-23.

15. Cicuttini F, Forbes A, Morris K, Darling S, Bailey M, Stuckey S. Gender differences in knee cartilage volume as measured by magnetic resonance imaging. Osteoarthr Cartil. 1999;7(3):265-71.

16. Cicuttini F, Wluka A, Wang Y, Stuckey S. The determinants of change in patella cartilage volume in osteoarthritic knees. J Rheumatol. 2002;29(12): 2615-9.

17. Hanna F, Ebeling PR, Wang Y, O'Sullivan R, Davis S, Wluka AE, Cicuttini FM. Factors influencing longitudinal change in knee cartilage volume measured from magnetic resonance imaging in healthy men. Ann Rheum Dis. 2005; 64(7):1038-42.

18. Wluka AE, Wolfe R, Davis SR, Stuckey S, Cicuttini FM. Tibial cartilage volume change in healthy postmenopausal women: a longitudinal study. Ann Rheum Dis. 2004:63(4):444-9.

19. Wluka AE, Stuckey S, Snaddon J, Cicuttini FM. The determinants of change in tibial cartilage volume in osteoarthritic knees. Arthritis Rheum. 2002:46(8): 2065-72.

20. Ding C, Martel-Pelletier J, Pelletier JP, Abram F, Raynauld JP, Cicuttini F, Jones $\mathrm{G}$. Two-year prospective longitudinal study exploring the factors 
associated with change in femoral cartilage volume in a cohort largely without knee radiographic osteoarthritis. Osteoarthr Cartil. 2008;16(4):443-9.

21. Ding C, Cicuttini F, Scott F, Cooley H, Jones G. Association between age and knee structural change: a cross sectional MRI based study. Ann Rheum Dis. 2005;64(4):549-55.

22. Srikanth VK, Fryer JL, Zhai G, Winzenberg TM, Hosmer D, Jones G. A metaanalysis of sex differences prevalence, incidence and severity of osteoarthritis. Osteoarthr Cartil. 2005;13(9):769-81.

23. Roman-Blas JA, Castaneda S, Largo R, Herrero-Beaumont G. Osteoarthritis associated with estrogen deficiency. Arthritis Res Ther. 2009;11(5):241.

24. Barrett-Connor E, Goodman-Gruen D. Gender differences in insulin-like growth factor and bone mineral density association in old age: the Rancho Bernardo Study. J Bone Miner Res. 1998;13(8):1343-9.

25. van der Kraan PM, Buma P, van Kuppevelt T, van den Berg WB. Interaction of chondrocytes, extracellular matrix and growth factors: relevance for articular cartilage tissue engineering. Osteoarthr Cartil. 2002;10(8):631-7.

26. Cotofana S, Buck R, Wirth W, Roemer F, Duryea J, Nevitt M, Eckstein F, Osteoarthritis Initiative Investigators G. Cartilage thickening in early radiographic knee osteoarthritis: a within-person, between-knee comparison. Arthritis Care Res (Hoboken). 2012;64(11):1681-90.

27. Frobell RB, Nevitt MC, Hudelmaier M, Wirth W, Wyman BT, Benichou O, Dreher D, Davies R, Lee JH, Baribaud F, et al. Femorotibial subchondral bone area and regional cartilage thickness: a cross-sectional description in healthy reference cases and various radiographic stages of osteoarthritis in 1,003 knees from the Osteoarthritis Initiative. Arthritis Care Res (Hoboken). 2010;62(11):1612-23.

28. Crema MD, Hunter DJ, Burstein D, Roemer FW, Li L, Eckstein F, Krishnan N, Hellio Le-Graverand MP, Guermazi A. Association of changes in delayed gadolinium-enhanced MRI of cartilage (dGEMRIC) with changes in cartilage thickness in the medial tibiofemoral compartment of the knee: a 2 year follow-up study using 3.0 T MRI. Ann Rheum Dis. 2014;73(11):1935-41.

29. Frobell RB. Change in cartilage thickness, posttraumatic bone marrow lesions, and joint fluid volumes after acute ACL disruption: a two-year prospective MRI study of sixty-one subjects. J Bone Joint Surg Am. 2011 93(12):1096-103.

30. Zheng $\mathrm{H}$, Chen C. Body mass index and risk of knee osteoarthritis: systematic review and meta-analysis of prospective studies. BMJ Open. 2015;5(12):e007568.

31. Mezhov V, Ciccutini FM, Hanna FS, Brennan SL, Wang YY, Urquhart DM, Wluka AE. Does obesity affect knee cartilage? A systematic review of magnetic resonance imaging data. Obes Rev. 2014;15(2):143-57.

32. Gersing AS, Schwaiger BJ, Nevitt MC, Joseph GB, Chanchek N, Guimaraes JB, Mbapte Wamba J, Facchetti L, McCulloch CE, Link TM. Is weight loss associated with less progression of changes in knee articular cartilage among obese and overweight patients as assessed with MR imaging over 48 months? Data from the Osteoarthritis Initiative. Radiology. 2017;284(2): 508-20.

33. Gersing AS, Schwaiger BJ, Nevitt MC, Zarnowski J, Joseph GB, Feuerriegel G, Jungmann PM, Guimaraes JB, Facchetti L, McCulloch CE, et al. Weight loss regimen in obese and overweight individuals is associated with reduced cartilage degeneration: 96-month data from the Osteoarthritis Initiative. Osteoarthr Cartil. 2019;27(6):863-70.

34. Gersing AS, Solka M, Joseph GB, Schwaiger BJ, Heilmeier U, Feuerriegel G, Nevitt MC, McCulloch CE, Link TM. Progression of cartilage degeneration and clinical symptoms in obese and overweight individuals is dependent on the amount of weight loss: 48-month data from the Osteoarthritis Initiative. Osteoarthr Cartil. 2016;24(7):1126-34.

35. Zhai G, Blizzard L, Srikanth V, Ding C, Cooley H, Cicuttini F, Jones G. Correlates of knee pain in older adults: Tasmanian Older Adult Cohort Study. Arthritis Rheum. 2006;55(2):264-71.

36. Jones $G$, Ding C, Scott F, Glisson M, Cicuttini F. Early radiographic osteoarthritis is associated with substantial changes in cartilage volume and tibial bone surface area in both males and females. Osteoarthr Cartil. 2004; 12(2):169-74.

37. Altman RD, Hochberg M, Murphy WA Jr, Wolfe F, Lequesne M. Atlas of individual radiographic features in osteoarthritis. Osteoarthr Cartil. 1995; 3(Suppl A):3-70.

38. Bellamy N, Buchanan WW, Goldsmith CH, Campbell J, Stitt LW. Validation study of WOMAC: a health status instrument for measuring clinically important patient relevant outcomes to antirheumatic drug therapy in patients with osteoarthritis of the hip or knee. J Rheumatol. 1988;15(12): 1833-40.
39. Tian J, Venn A, Otahal P, Gall S. The association between quitting smoking and weight gain: a systematic review and meta-analysis of prospective cohort studies. Obes Rev. 2015;16(10):883-901.

40. Dore DA, Winzenberg TM, Ding C, Otahal P, Pelletier JP, Martel-Pelletier J, Cicuttini FM, Jones $\mathrm{G}$. The association between objectively measured physical activity and knee structural change using MRI. Ann Rheum Dis. 2013;72(7):1170-5

41. Teichtahl AJ, Wluka AE, Tanamas SK, Wang Y, Strauss BJ, Proietto J, Dixon JB, Jones G, Forbes A, Cicuttini FM. Weight change and change in tibial cartilage volume and symptoms in obese adults. Ann Rheum Dis. 2015; 74(6):1024-9.

42. Antony B, Ding C, Stannus O, Cicuttini F, Jones G. Association of baseline knee bone size, cartilage volume, and body mass index with knee cartilage loss over time: a longitudinal study in younger or middle-aged adults. J Rheumatol. 2011;38(9):1973-80.

43. Hunter DJ, Zhang YQ, Tu X, Lavalley M, Niu JB, Amin S, Guermazi A, Genant $H$, Gale D, Felson DT. Change in joint space width: hyaline articular cartilage loss or alteration in meniscus? Arthritis Rheum. 2006;54(8):2488-95.

44. Ding C, Cicuttini F, Blizzard L, Jones G. Genetic mechanisms of knee osteoarthritis: a population-based longitudinal study. Arthritis Res Ther. 2006:8(1):R8.

45. Shultz SJ, Nguyen AD, Schmitz RJ. Differences in lower extremity anatomical and postural characteristics in males and females between maturation groups. J Orthopaedic Sports Phys Ther. 2008;38(3):137-49.

46. Eckstein F, Wirth W, Hudelmaier M, Stein V, Lengfelder V, Cahue S, Marshall M, Prasad P, Sharma L. Patterns of femorotibial cartilage loss in knees with neutral, varus, and valgus alignment. Arthritis Rheum. 2008;59(11):1563-70.

47. Cicuttini F, Wluka A, Hankin J, Wang Y. Longitudinal study of the relationship between knee angle and tibiofemoral cartilage volume in subjects with knee osteoarthritis. Rheumatol. 2004;43(3):321-4.

48. Oliveria SA, Felson DT, Reed Jl, Cirillo PA, Walker AM. Incidence of symptomatic hand, hip, and knee osteoarthritis among patients in a health maintenance organization. Arthritis Rheum. 1995;38(8):1134-41.

49. Ding C, Cicuttini F, Scott F, Cooley H, Boon C, Jones G. Natural history of knee cartilage defects and factors affecting change. Arch Intern Med. 2006; 166(6):651-8.

50. Ostrowska M, Maslinski W, Prochorec-Sobieszek M, Nieciecki M, SudolSzopinska I. Cartilage and bone damage in rheumatoid arthritis. Reumatologia. 2018;56(2):111-20.

51. Anandacoomarasamy A, Leibman S, Smith G, Caterson I, Giuffre B, Fransen M, Sambrook PN, March L. Weight loss in obese people has structuremodifying effects on medial but not on lateral knee articular cartilage. Ann Rheum Dis. 2012;71(1):26-32.

52. Cicuttini FM, Wluka AE, Wang Y, Stuckey SL. Longitudinal study of changes in tibial and femoral cartilage in knee osteoarthritis. Arthritis Rheum. 2004; 50(1):94-7.

53. Bruynesteyn K, Van Der Heijde D, Boers M, Saudan A, Peloso P, Paulus H, Houben H, Griffiths B, Edmonds J, Bresnihan B, et al. Detecting radiological changes in rheumatoid arthritis that are considered important by clinical experts: influence of reading with or without known sequence. J Rheumatol. 2002;29(11):2306-12.

54. Ross PD, Huang C, Karpf D, Lydick E, Coel M, Hirsch L, Wasnich RD. Blinded reading of radiographs increases the frequency of errors in vertebral fracture detection. J Bone Miner Res. 1996;11(11):1793-800

\section{Publisher's Note}

Springer Nature remains neutral with regard to jurisdictional claims in published maps and institutional affiliations. 